\title{
Using convex preference cones in multiple criteria decision making and related fields
}

\author{
Nasim Nasrabadi ${ }^{1}$ - Akram Dehnokhalaji ${ }^{2}$ - Pekka Korhonen ${ }^{3} \cdot$ Jyrki Wallenius $^{3}$
}

Published online: 26 June 2019

(C) The Author(s) 2019

\begin{abstract}
This paper reviews our own and colleagues' research on using convex preference cones in multiple criteria decision making and related fields. The original paper by Korhonen, Wallenius, and Zionts was published in Management Science in 1984. We first present the underlying theory, concepts, and method. Then we discuss applications of the theory, particularly for finding the most preferred alternative, finding a partial and total rank ordering of alternatives, as well as developing algorithms for solving multi-objective integer and other optimization problems.
\end{abstract}

Keywords Multiple criteria decision making · Discrete $\cdot$ Convex preference cones . Data envelopment analysis · Integer programming

\section{JEL Classification C6}

\section{Introduction}

This paper is about multiple criteria decision making (MCDM). MCDM has been an active research field for more than half a century. We focus on problems in which the number of alternatives is countable. The number of alternatives can be either finite or countably infinite. Typical examples of the first type of problems are so-called evaluation problems, in which we assume that the set of all decision alternatives is explicitly known. We also discuss multi-objective integer programming problems, which are sometimes countably infinite. In evaluation problems we assume that the goal is either

Jyrki Wallenius

jyrki.wallenius@aalto.fi

1 Department of Mathematics, Faculty of Mathematical Science and Statistics, University of Birjand, Birjand, Iran

2 Operations and Information Management Department, Aston Business School, Aston University, Birmingham B4 7ET, UK

3 Department of Information and Service Management, Aalto University School of Business, Helsinki, Finland 
to find the most preferred alternative (from the decision-maker's point of view), a small subset of good alternatives, or rank order the alternatives from best to worst. We do not assume explicit knowledge of the decision-maker's underlying value function, but we assume the value function to be quasi-concave and increasing. This corresponds to convex-to-the-origin indifference contours, a rather common assumption in economics.

More specifically, our paper summarizes our work and our colleagues' work on convex preference cones, a stream of research started by Pekka Korhonen, Jyrki Wallenius, and Stanley Zionts in early 1980s. Their first publication dealing with this topic emerged in 1984, although the work was started during 1980. Over the years we have collaborated with many colleagues on convex preference cones. Roughly a dozen publications are joint with Murat Köksalan, who completed his Ph.D. at State University of New York at Buffalo in 1983 under the supervision of Mark Karwan and Stanley Zionts. His dissertation topic was convex preference cones. Murat Köksalan has since worked with many Turkish Ph.D. students and colleagues on this topic. In 2006, while Jyrki Wallenius was a Visiting Professor at Arizona State University, he suggested the use of convex preference cones in Evolutionary Multi-objective Optimization, a new and active field of research, as a dissertation topic to a student (Marquis). Their collaboration resulted in two joint publications. Akram Dehnokhalaji and Nasim Nasrabadi, at the time Ph.D. students from Iran, joined the Korhonen-Wallenius team around 2009. One of the ideas was to generate a partial order and a total order with the help of convex preference cones. The collaboration with Banu Lokman from METU has resulted in two publications dealing with multi-objective integer programming problems, with the help of convex preference cones. One of the papers develops an exact algorithm for solving the problem, a more recent paper a powerful heuristic based on approximate cones. Moreover, Korhonen and Wallenius have collaborated with Majid Soleimani on the mathematical aspects of convex preference cones. An interesting paper reviewing the use of convex cones is Karsu (2013). However, our review is more comprehensive and up-to-date. Olson (1996) also provided decision aids for selection problems.

The idea of convex preference cones is relatively simple. Assume we want to find the most preferred alternative from among a (large) set of alternatives. Each alternative is evaluated with multiple criteria. The most preferred alternative depends on the decision-maker's preferences. How can we reliably and easily capture the decision-maker's preferences? Multiple approaches have been suggested in the literature. We have always liked pairwise comparisons. There is some behavioral research, which indicates that such comparisons are relatively easy. However, to identify the most preferred alternative with the help of pairwise comparisons may require a prohibitive number of such comparisons. We must therefore find mechanisms, which would allow us to eliminate many alternatives, which obviously are not of interest to the decision-maker. The convex preference cones are one such mechanism. Let us illustrate with an example. Let us assume that we simply randomly ask the decision-maker to make a number of pairwise comparisons. Assume, for example, that $\mathrm{A}$ is preferred to $\mathrm{B}, \mathrm{C}$ is preferred to $\mathrm{D}$, and $\mathrm{E}$ is preferred to $\mathrm{D}$. Next, group the alternatives into sets, where we know the worst alternative (in the set). This results in two sets: (1) A and B (B worst), (2) C, D, and E (D worst). In both cases connect the points from the best to the worst (A to B; C to D and $\mathrm{E}$ to $\mathrm{D}$ ) and extend the lines beyond the worst points, i.e. the dotted lines with the arrows stand for the spanning 
directions of the cones. The result is two preference cones: one two-point cone (Fig. 1a) and one (yellow or shaded) three-point cone (Fig. 1b). We have proved that all alternatives which lie in the cones or below them, are not better than the known worst alternative - and can be eliminated from further consideration. The proof is based on assuming an increasing quasi-concave value function.

The structure of our paper is as follows. Section 2 presents the basic concepts and theory of convex preference cones. Section 3 presents the original convex preference cone method and enhancements for finding the most preferred alternative. Section 4 reviews applications of the convex cone theory to discrete problems. Section 5 discusses applications to solving integer and continuous optimization problems. Section 6 concludes the paper.

\section{Basic concepts and theory}

In this section we explain the basic concepts and theory of convex preference cones, which are utilized in solving different types of MCDM problems (Korhonen et al. 1984). We first illustrate the main idea by using a simple example and then generalize it by presenting some theoretical results. Without loss of generality, we assume in the sequel that more is better for each criterion. The value function is assumed to be increasing and quasi-concave. Also see Hazen (1983) and Ramesh et al. (1988).

Example 1 Assume that we have a discrete Bi-criteria decision making problem. Suppose that we do not explicitly know the decision-maker's (DM) value function, but assume that it is increasing and quasi-concave (the corresponding definitions will be provided later). Moreover, she/he has expressed preferences in the form of pairwise comparisons. For simplicity, assume that there are only five alternatives denoted by $S=\{A, B, C, D, E\}$, and the DM prefers alternative $B$ to $D$. The set of alternatives in the criterion space ( $R^{2}$-space) is presented in Fig. 2.

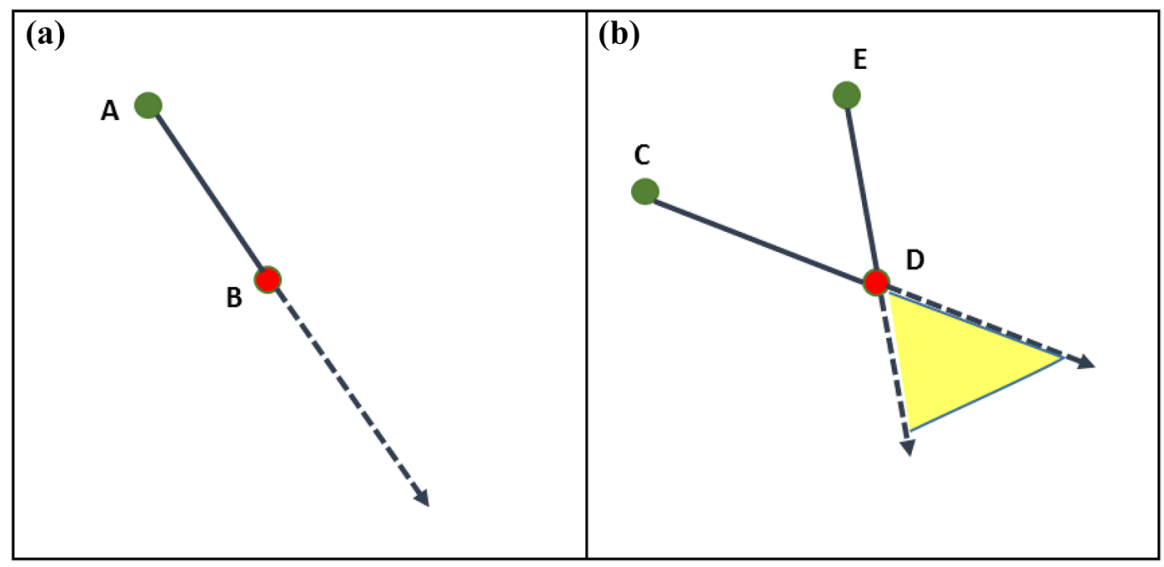

Fig. 1 Illustration of convex cones: a two-point cone, $\mathbf{b}$ three-point cone 


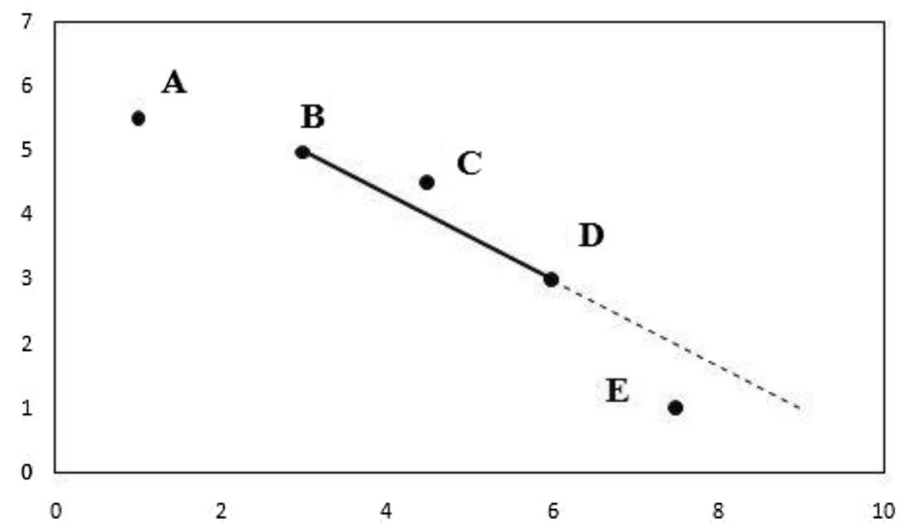

Fig. 2 Set of five alternatives in Example 1

Based on the main properties of the value function, i.e. being increasing and quasi-concave, we are able to extract some additional information about some of the other alternatives (besides $B$ and $D$ ). In brief, the following statements hold true:

1. Each alternative located on the line segment $B D$ or above it is at least as preferred as $D$.

2. Alternative $D$ is at least as preferred as each alternative located on or below the half-line (=two-point cone) emanating from $D$ in the direction $\overrightarrow{B D}$.

Hence, alternative $C$ is at least as preferred as $D$, whereas $D$ is at least as preferred as $E$. However, we are unable to make preference statements regarding alternative $A$, since it satisfies neither (1) nor (2).

Now, in order to generalize the idea, we provide some definitions. Assume that we have $m$ alternatives to be evaluated by $p$ criteria $(p \geq 2)$. We denote this set of alternatives by $S=\left\{X_{1}, \ldots, X_{m}\right\}$ where $X_{i}=\left(x_{i 1}, \ldots, x_{i p}\right)^{T} \in R^{p}$ is the $i$-th alternative. The main idea of the "convex cone approach" is to construct preference cones based on the DM's pairwise comparisons. Utilizing the properties of the underlying cones enables us to extract additional preferences regarding other alternatives. For the theory to work, the DM's value function is assumed to be increasing and quasiconcave. In what follows, we provide the necessary definitions.

Definition 1 Assume that $X, Y \in R^{p}$. Then $X$ dominates $Y$ if and only if $X \geq Y$ and $X \neq Y$. Moreover, a vector $X^{*} \in R^{p}$ in a given set $S \subseteq R^{p}$ is said to be non-dominated if and only if there does not exist another vector in $S$ which dominates $X^{*}$.

To illustrate Definition 1, consider the set $S=\{A, B, C, D, E\}$ as shown in Fig. 2. It is easy to verify that all alternatives in set $S$ are non-dominated, because for each $X \in S$ there are no other members in $S$, which dominate $X$. 
Definition 2 A function $f: R^{p} \rightarrow R$ is called a value function if it has the following properties:

1. $f\left(X^{*}\right)>f(X)$, if $X^{*}$ dominates $X$.

2. $f\left(X^{*}\right)>f(X)$, if and only if $X^{*}$ is preferred to $X$.

3. $f\left(X^{*}\right) \geq f(X)$, if $X^{*}$ is at least as preferred as $X$.

Part (1) means that $f$ is increasing, whereas parts (2) and (3) clarify the concept of preference with respect to a given value function. Formally, we write $X^{*}>X$, if $X^{*}$ is preferred to $X$, and $X^{*} \geqslant X$ if $X^{*}$ is at least as preferred as $X$.

Definition 3 A function $f: \Omega \subseteq R^{p} \rightarrow R$ on a non-empty convex set $\Omega$ is quasiconcave, if $f(\lambda X+(1-\lambda) Y) \geq \min \{f(X), f(Y)\}$, for all $X, Y \in \Omega$ and $\lambda \in[0,1]$.

Note that quasi-concave value functions correspond to convex-to-the origin indifference contours.

If the value function is explicitly known, it is straightforward to solve the MCDM problem, given certain regularity conditions. Unfortunately, in most realworld problems, the underlying value function is not explicitly known. However, it can be assumed that the DM's value function satisfies some pre-known properties, such as monotonicity, linearity, concavity, quasi-concavity, etc. In order to implement the "convex cone" approach, the underlying value function is assumed to be increasing and quasi-concave. As mentioned in Korhonen et al. (1984): "The quasi-concave functions are the most general class of functions for which the theory needed in the convex cone approach is valid." In addition, when implementing the convex cone approach, it is assumed that the DM is provided with some preference information regarding the set of alternatives in the form of pairwise comparisons. Then based on this preference information and using the main properties of convex cones, it is possible to extract additional preference information concerning many of the alternatives.

Assume that the DM's preferences are given as

$$
P=\left\{\left(X_{r}, X_{s}\right) \mid X_{r}>X_{s}, 1 \leq r, s \leq m\right\} .
$$

In fact, set $P$ provides the preference information for some pairs of alternatives in $S$ in the form of pairwise comparisons. Regarding each subset of $S$, in which the least preferred alternative is given, a convex cone can be constructed with the property that the least preferred alternative is the vertex and the other alternative(s) are generator(s) of the cone. Formally, we define a preference subset, a preference cone, and a preference polyhedron as follows.

Definition 4 Let $X_{1}, \ldots, X_{k-1}, X_{k}, \ldots, X_{q} \in S$ be $q$ distinct points with the property that $X_{i}>X_{k}$ for $i=1, \ldots, q$ and $i \neq k$. 
1. The set including alternatives $X_{1}, \ldots, X_{k-1}, X_{k}, \ldots, X_{q}$ is called a preference subset of $P$ and is denoted by $\left\{X_{1}, \ldots, X_{q} ; X_{k}\right\}$, where $X_{k}$ is the least preferred alternative in the set.

2. The cone with vertex $X_{k}$ is defined as

$$
C\left(X_{1}, \ldots, X_{q} ; X_{k}\right)=\left\{Z \mid Z=X_{k}+\sum_{i=1, i \neq k}^{q} \mu_{i}\left(X_{k}-X_{i}\right), \quad \mu_{i} \geq 0, \quad i=1, \ldots, q, \quad i \neq k\right\}
$$

and is called a preference cone.

3. The polyhedron spanned by points $X_{1}, \ldots, X_{q}$ is defined as

$$
H\left(X_{1}, \ldots, X_{q} ; X_{k}\right)=\left\{Y \mid Y=\sum_{i=1}^{q} \lambda_{i} X_{i}, \sum_{i=1}^{q} \lambda_{i}=1, \lambda_{i} \geq 0, i=1, \ldots, q\right\}
$$

and is called a preference polyhedron.

Using Theorem 1, we are able to detect additional inferior and superior alternatives (to $X_{k}$ ), without explicitly asking about them. For a detailed proof, see Korhonen et al. (1984).

Theorem 1 Let $f: R^{p} \rightarrow R$ be a quasi-concave value function and $\left\{X_{1}, \ldots, X_{q} ; X_{k}\right\}$ be a preference subset. Then:

1. For each $Z \in C\left(X_{1}, \ldots, X_{q} ; X_{k}\right)$, we have $X_{k}>Z$,

2. For each $Y \in H\left(X_{1}, \ldots, X_{q} ; X_{k}\right)$, we have $Y \geqslant X_{k}$.

In other words, if an alternative lies in or below the convex cone $C$, it is inferior or indifferent to $X_{k}(1)$. Moreover, if an alternative lies in the polyhedron $H$ or above it, it is superior or indifferent to $X_{k}$ (2). (See Korhonen et al. (2017) for proofs of some additional properties of quasi-concave value functions and convex cones.)

Korhonen et al. (1984) provide simple LP formulations to check whether conditions in (1) and/or (2) are met.

Example 2 Consider the discrete Bi-criteria decision problem with five non-dominated alternatives $S=\{A, B, C, D, E\}$ presented in Fig. 2. Assume the DM prefers alternative $B$ to $D$. This gives us the preference subset $\{B, D ; D\}$. Based on Definition 1 , the corresponding preference cone is formed as the half-line emanating from $D$ in the direction $\overrightarrow{B D}$ and the preference polyhedron is given as the line segment $B D$. Now, based on Theorem 1, it is easy to verify that $C$ is preferred to $D$, and $D$ is preferred to $E$.

Although Theorem 1 provides useful information about preference cones and preference polyhedra, we mainly focus on using convex cones, i.e. on part (1) of Theorem 1 in this paper. Based on part (1) of the theorem, the convex cone 
$C\left(X_{1}, \ldots, X_{q} ; X_{k}\right)$ and the region dominated by it, denoted by $C^{-}\left(X_{1}, \ldots, X_{q} ; X_{k}\right)$, is considered an inferior region and can be discarded from further consideration. Hence for each alternative, the idea is to check if it is included in such a region or not. In the following we operationalize the idea.

Suppose that $X_{o}$ is a given alternative such that $X_{o} \notin\left\{X_{1}, \ldots, X_{q} ; X_{k}\right\}$, and our aim is to check the preference status of $X_{o}$ compared to $X_{k}$. To do this, it is enough to check whether $X_{o}$ belongs to the cone-dominated region or not. The following LP problem was formulated by Korhonen et al. (1984):

$$
\begin{aligned}
& \varepsilon^{*}=\max \varepsilon \\
& \text { s.t. } \quad \sum_{i=1, i \neq k}^{q} \mu_{i}\left(X_{k}-X_{i}\right)-\varepsilon 1 \geq X_{0}-X_{k} \\
& \mu_{i} \geq 0, i=1, \ldots, q, i \neq k,
\end{aligned}
$$

where $1=(1, \ldots, 1)$. Based on model (4) and part (1) of Theorem 1, it can be easily verified that if $\varepsilon^{*}>0$, then $X_{k}$ is preferred to $X_{0}$. Moreover, if $\varepsilon^{*}=0$, then $X_{k}$ is at least as preferred as $X_{o}$. It is worthwhile to note that some other helpful results can also be obtained from the above problem. The reader is referred to Korhonen et al. (2016) for a comprehensive discussion.

The reader may ask, how do we know, whether the decision-maker has a quasiconcave value function or not. Given set $P$ of the DM's preferences, Korhonen et al. (1986) developed a simple approach, which checks whether there exists a quasi-concave value function consistent with the DM's preferences. Their approach investigates this by solving the following linear program:

$$
\begin{aligned}
& \varepsilon^{*}=\max \varepsilon \\
& \text { s.t. } \quad \lambda(Y)^{T}(X-Y) \geq \varepsilon,(X, Y) \in P \\
& 1^{T} \lambda(Y)=1, \lambda(Y) \geq 0, \forall Y, P(Y) \neq \emptyset
\end{aligned}
$$

where $P(Y)=\{X \mid(X, Y) \in P\}$. At optimality, if $\varepsilon^{*} \geq 0$, it is concluded that the DM's value function is quasi-concave, or equivalently, the assumption of quasi-concavity is consistent with the DM's preferences. In other words, we do not reject the hypothesis that the value function is quasi-concave. Note that the condition is necessary, but not sufficient. Köksalan and Sagala (1995) have developed an efficient approach that quickly identifies whether the DM's preferences are consistent with a linear, quasi-concave, or a more general function.

In Fig. 3a, b, we illustrate our considerations by comparing three alternatives $\mathrm{A}, \mathrm{B}$, and $\mathrm{C}$ in two dimensions. The values of the alternatives in the criterion space (criteria to be maximized) are A: $(1,2.5), B:(2,2)$, and $C:(2.5,1)$.

In Fig. 3a, we assume that $B>A$ and $B>C$. In this case, we can see that it possible to find vector $\lambda$ such that its scalar product between vectors $\overrightarrow{C B}$ and $\overrightarrow{A B}$ is greater than zero. It means that there is no reason to reject the assumption that the value function is quasi-concave, whereas such a vector cannot be found in case b, and we reject the quasi-concavity assumption. 
Possible Quasi-Concave Value Function

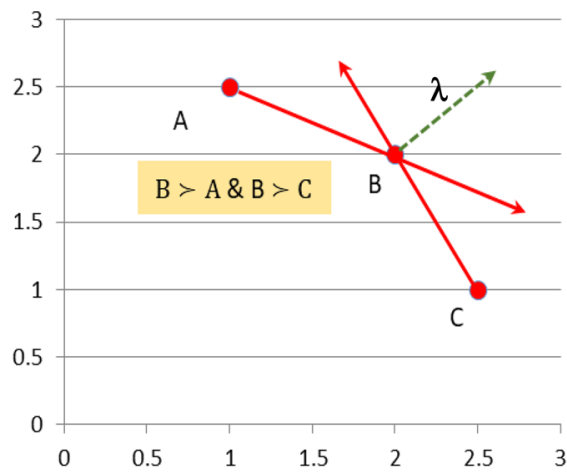

No Quasi-Concave Value Function

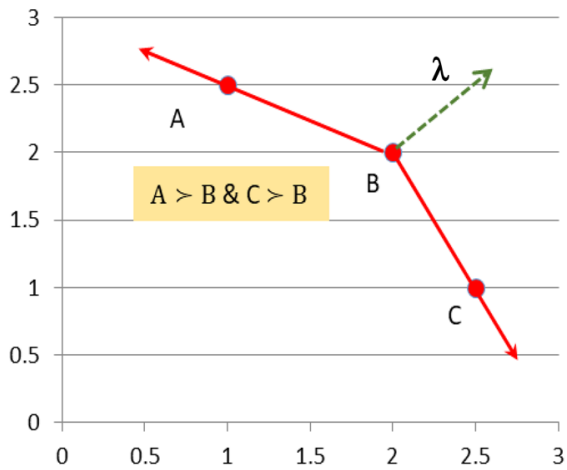

Fig. 3 Illustration of quasi-concavity test

\subsection{Dual cone approach}

In case the number of alternatives included in the preference subset is large compared to the number of criteria, i.e. $q>p$, it is helpful to use a dual cone approach instead of solving model (4). This issue was investigated in Korhonen et al. (2016). To illustrate the dual cone approach, we first provide the following definition.

Definition 5 The polyhedral cone $W$, associated with the preference subset $\left\{X_{1}, \ldots, X_{q} ; X_{k}\right\}$ is defined as $W=\left\{V \in R^{p} \mid V\left(X_{i}-X_{k}\right) \geq 0, i=1, \ldots, q, V \geq 0\right\}$.

Geometrically, $W$ is the set of all non-negative vectors $V$ with the property that the angle between $V$ and all vectors of the form $X_{i}-X_{k}$ for all $i=1, \ldots, q, \quad i \neq k$ is between zero and $\frac{\pi}{2}$. Now, assume that $V_{1}, \ldots, V_{t}$ are the extreme generators of $W$. The following theorem clarifies the relation between the polyhedral cone and our original model (Korhonen et al. 2016).

Theorem 2 Considering above notations,

1. $\varepsilon^{*} \geq 0$ if and only if $V_{i}\left(X_{k}-X_{o}\right) \geq 0$ for $i=1, \ldots, t$. Equivalently, $X_{k}$ is at least as preferred as $X_{o}$

2. $\varepsilon^{*}>0$ if and only if $V_{i}\left(X_{k}-X_{o}\right)>0$ for $i=1, \ldots, t$. Equivalently, $X_{k}$ is preferred to $X_{o}$

Theorem 2 enables us to check the preference status of $X_{o}$ in comparison with $X_{k}$, by means of extreme generators of $W$. To simplify the idea, notice that if $V_{i}\left(X_{k}-X_{o}\right) \geq 0$ for $i=1, \ldots, t$, then $X_{k}$ is at least as preferred as $X_{o}$. Moreover, if $V_{i}\left(X_{k}-X_{o}\right)>0$ for $i=1, \ldots, t$, then $X_{k}$ is preferred to $X_{o}$. This result forms the fundamental idea of the dual cone approach. 
Example 3 Let $X_{1}=(2.5,3.5)^{T}$ and $X_{k}=(4,2)^{T}$. Assume that $X_{1}>X_{k}$ and we aim to check the preference status of $X_{p}=(4.5,1)^{T}$ and $X_{q}=(5,1.5)^{T}$ with respect to the preference cone $C\left(X_{1}, X_{k} ; X_{k}\right)$. From Fig. $4 \mathrm{a}$ it can be observed that $X_{k}$ is preferred to $X_{p}$, however no preference information is derived for $X_{q}$. The same information can be derived using the dual cone. The cone $W$ is represented in Fig. $4 \mathrm{~b}$ with $V_{1}=(0,1)^{T}$ and $V_{2}=(1,1)^{T}$ as its extreme generators. Moreover, the vectors $X_{k}-X_{p}$ and $X_{k}-X_{q}$ are also depicted. We can see that the angle between $X_{k}-X_{p}$ and both $V_{1}$ and $V_{2}$ belongs to $\left(0, \frac{\pi}{2}\right)$, which implies that $\varepsilon^{*}>0$. However, the angle between $X_{k}-X_{q}$ and $V_{2}$ is in $\left(\frac{\pi}{2}, \pi\right)$ for $X_{q}$, and hence $\varepsilon^{*}<0$.

\section{The original method and its enhancements}

Based on the original theory provided in the previous section, a number of studies have been conducted to enhance it. In this section we review a number of such studies. All of them are based on Korhonen et al. (1984). A quasi-concave value function is assumed in each case. Pairwise comparisons are the mechanism of eliciting preference information from the DM.

\subsection{The original approach: Korhonen et al. (1984)}

The original article developed the convex cone theory and an operational approach for identifying the most preferred alternative among a set of discrete alternatives. The original approach works as follows. To begin with, a linear value function with arbitrary weights is generated and the alternative which maximizes this value function is determined. Then the DM is asked to compare this alternative with all of its adjacent alternatives in a pairwise fashion, in the spirit of the Zionts and Wallenius algorithm (Zionts and Wallenius 1976). Based on the obtained preference information among the alternatives, all possible convex cones are generated. Then, by applying model (4) (or its dual form), all alternatives located in the cone-dominated regions are identified and eliminated. Moreover, the DM's pairwise comparisons are used to update the underlying weights of the linear value function. With the new set of consistent weights, the procedure is repeated until all alternatives except one are

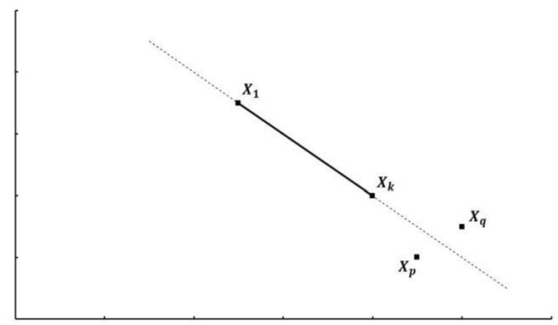

(a) Alternatives in original space

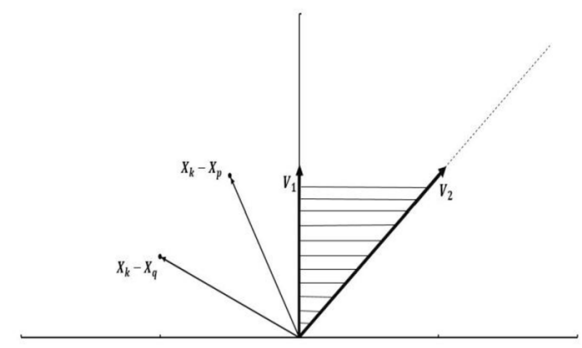

(b) Extreme generators of the dual cone

Fig. 4 The dual cone for Example 1 
eliminated. The remaining alternative is presented to the DM as the most preferred. The authors report on some computational statistics and numbers of pairwise comparisons required to identify the most preferred alternative for problems of different sizes. The results were encouraging.

\subsection{Introducing dummy alternatives: Köksalan et al. (1984) and Köksalan and Taner (1992)}

As part of Köksalan's Ph.D. research, he introduced improvements to the original algorithm, published in Köksalan et al. (1984). The main idea for improvement was based on introducing dummy alternatives. The authors believed that by introducing dummy alternatives in a suitable way, it was possible to eliminate more alternatives in each step of the algorithm. Dummy alternatives chosen smartly can expand the cone dominated region and therefore more alternatives are likely to be eliminated. The original dummies were convex combinations of $X_{k}$ and alternatives found better than it (in pairwise comparisons). Finally, they compared their version of the algorithm with the original version of Korhonen et al. (1984). The performance measure was the total number of pairwise comparisons required to solving the problem (identifying the most preferred alternative). The results of computer simulations showed that for almost all test problems, the improved algorithm performed better than the original algorithm. Also, it was verified that by implementing a heuristic stopping rule, in general the most preferred alternative was found in less than 20 pairwise comparisons.

Köksalan and Taner (1992) developed an improved version of Köksalan et al. (1984) regarding the rule for selecting the dummy alternatives. They suggested using as dummies alternatives, which dominate the least preferred of the cone generators and which are less preferred than the remaining cone generators. The purpose of the improvements was to reduce the number of pairwise comparisons required from the DM. Moreover, they suggested that by using three-point cones along with dummy alternatives, the algorithm would perform more effectively. Figure 5 illustrates the use of dummy variables, denoted $X_{d}$. In the left-hand figure, we assume that $X_{1}$ is preferred to $X_{d}$. In the right-hand figure, $X_{d}$ is preferred to $X_{k}$.

In an early paper, Malakooti (1988) also presented different ways to select the cone generators, including the use of dummy alternatives to increase the area that can be eliminated by the cones. He also discussed the use of trade-off information to eliminate less preferred alternatives and find better ones.
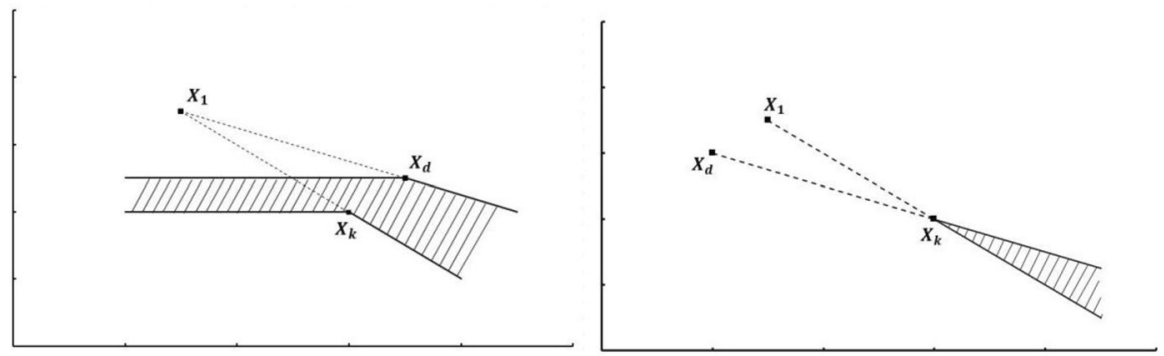

Fig. 5 Using dummy alternatives to enlarge the cone dominated region 


\subsection{Techniques for minimizing number of pairwise comparisons: Taner and Köksalan (1991) and Özpeynirci et al. (2017)}

One of the most important concerns in implementing interactive approaches to finding the most preferred alternative is the amount of preference information required from the DM. Taner and Köksalan (1991) investigated this issue in the framework of the convex cone approach. They conducted several experiments to investigate how some factors, such as the number of cone generators, the way in which the generators are selected, and the order of making pairwise comparisons affect the total number of pairwise comparisons. They observed that cones having three generators are generally more powerful than other cones in the first iterations, when the number of alternatives exceeds 10 . However, two-point cones guarantee to eliminate at least one alternative at each iteration, in case we have 10 or fewer alternatives. Therefore,

using both types of cones, three-point cones for the first iterations and two-point cones for the remaining iterations, is suggested.

In a recent paper, Özpeynirci et al. (2017) revisited the issue of minimizing the number of questions asked of the DM. They significantly enhanced the original convex cone idea by systematically choosing the pairs of alternatives for comparison. The idea was based on the expected number of eliminated alternatives per pair. This depended on the estimated likelihood one alternative is preferred to another. The likelihood information is updated for not yet chosen pairwise alternatives. The rest of the algorithm follows the convex cone logic. The goal of the algorithm is to ask as few pairwise comparisons as possible.

\subsection{Ordinal, cardinal, and mixed criteria: Köksalan et al. $(1986,1988)$}

Köksalan et al. (1986) addressed the problem of finding the most preferred alternative among a set of finite alternatives, which are evaluated in terms of ordinal and cardinal criteria simultaneously. Moreover, a heuristic method to solve the problem with only cardinal criteria was also proposed. Experimental tests of the proposed algorithms indicate that for problems consisting of 100 and 150 alternatives with four criteria, the number of pairwise comparisons required to identify the most preferred alternative is approximately 20. Köksalan et al. (1988) paper deals with the case of pure ordinal criteria.

\section{Application of convex preference cones to discrete problems}

Most of the applications of the convex cone theory have been to discrete problems. We review a number of such studies. They include partitioning alternatives into acceptable and unacceptable; characterizing regions of "surely" and "possibly better" alternatives; ranking of alternatives; Evolutionary Multi-Objective Optimization; and Data Envelopment Analysis (DEA). 


\subsection{Partitioning alternatives into acceptable and unacceptable: Ulu and Köksalan (2001)}

Ulu and Köksalan (2001) considered the problem, which partitions alternatives into acceptable and unacceptable sets. Assuming that the DM's value function is linear, quasi-concave or general monotone, they developed algorithms to find an acceptable subset of alternatives. Their approach is of critical importance, especially when the number of alternatives is large. They conducted experimental tests to verify the validity of their proposed approach. Moreover, they used their approach in a real-world application with the purpose of admitting students to Middle East Technical University, Turkey. The previous idea has been generalized to any number of classes in Ulu and Köksalan (2014). They refer to the problem as sorting.

\subsection{Surely and possibly preferred alternatives: Korhonen et al. (1986)}

Assuming that a quasi-concave value function consistent with $P$ exists, Korhonen et al. (1986) defined and characterized the regions of "surely better solutions" and "possibly better solutions", when considering augmenting the original set of alternatives. The idea underlying this distinction is based on dominance and cone dominance. Assume that $A$ is the best alternative found so far. Then obviously, alternatives which dominate the most preferred alternative (so far), are surely better. In other words, the process that is used for finding the most preferred alternative so far, produces preference information (convex preference cones), which we can use to eliminate inferior alternatives from further consideration. The alternatives, which belong to the cone-dominated region, are surely less preferred than the most preferred alternative found so far. The region which is not surely better nor surely worse, consists of possibly better alternatives. The distinction between surely and possibly better alternatives is important when forming a total or partial rank order. Also see Karakaya and Köksalan (2018).

\subsection{Ranking}

Ranking is a problem that has interested decision scientists for quite some time. In the two papers outlined below (Dehnokhalaji et al. 2011, 2014), we use the convex cone idea to generate a partial order and a total order of a finite set of alternatives. Ranking has many possible applications. It can, among others, be used to incorporate preference information in, e.g., Data Envelopment Analysis (DEA) and Evolutionary Multi-Objective Optimization (EMO). Both applications are briefly described below. A related problem is to find the k-best alternatives and rank them. This problem has been addressed by Köksalan (1989). 


\subsubsection{Partial order: Dehnokhalaji et al. (2011)}

In this paper, we considered the problem of finding a preference-based strict partial order for a finite set of multiple criteria alternatives. We developed an approach based on information provided by the decision maker in the form of pairwise comparisons. We assumed that the decision-maker's value function is not explicitly known, but it has a quasi-concave form. Based on this assumption, we constructed convex cones providing additional preference information to partially order the set of alternatives. We also extended the information obtained from the quasi-concavity of the value function to derive heuristic information that enriches the strict partial order.

\subsubsection{Total order: Dehnokhalaji et al. (2014)}

The problem of finding a strict total order for a finite set of multiple criteria alternatives was considered. Our research extended previous work by us, which considered finding a partial order for a finite set of alternatives. We merged the preference information extracted from the preference cones and corresponding polyhedral sets, with the information derived from pairwise comparisons of two alternatives, yielding a preference matrix. This preference matrix was used as input to an integer programming model to obtain a strict total order that provides a transitive ranking for the set of alternatives.

\subsection{Evolutionary multi-objective optimization (EMO)}

Evolutionary multi-objective optimization (EMO) is a relatively new field, which uses concepts of survival of the fittest to solve complex optimization problems (Deb 2001). It started with the engineering community, and the goal was to generate the Pareto-optimal front for Bi-criteria problems. No decision maker was involved. EMO differs from classical optimization in many ways. Classical optimization algorithms typically seek to find an improving sequence of solutions, which hopefully converges to the optimal solution. EMO algorithms, on the other hand, operate with a set of candidate solutions, which we seek to improve over multiple (typically hundreds) of iterations. In EMO algorithms the fitness function is used to measure the quality of the set of candidate solutions.

\subsubsection{Fowler et al. (2010) and Marquis et al. (2015)}

Fowler et al. (2010) present a hybrid MCDM/EMO approach to interactively solve multi-objective optimization problems. A DM is involved and guides the search. The Fowler et al. algorithm uses a partial preference order, obtained via convex preference cones, to replace the fitness function in an EMO algorithm. We periodically ask the DM to make pairwise preference comparisons and use the resulting 
preference information to form convex preference cones. The cones allow us to rank solutions that the DM has not even considered. As before, we assume that the preference function is quasi-concave.

The above algorithm opens a number of behavioral questions. For example, what is the role of human inconsistencies on the convergence of the algorithm? How should the interactions be organized? More questioning early on or later on? In a relatively recent paper (Marquis et al. 2015) we investigated such questions. As test problems we used two- to four-objective combinatorial optimization problems, which are notoriously hard to solve. In the tests, two different preference functions were used: linear and Chebyshev. We concluded that it is possible to obtain solutions that are very good or even nearly optimal with a reasonable number of interactions.

\subsection{DEA/VEA}

Data envelopment analysis (DEA) is a linear programming-based technique for evaluating the relative efficiency of a number of homogenous decision-making units using multiple inputs to produce multiple outputs. In DEA the efficiency score is calculated for each unit in such a way that the units with efficiency score equal to one are diagnosed as efficient and the other units as inefficient. The original reference is Charnes et al. (1978). An important feature of DEA is that the original DEA model is value-free.

\subsubsection{Halme et al. (2014)}

This work builds on value efficiency analysis (VEA) proposed by Halme et al. (1999). Recall that the main motivation of value efficiency analysis (VEA) was to incorporate the decision-maker's preferences into DEA. The original VEA model assumes that the DM provides his/her preferences via determining the most preferred solution. In the original formulation this solution could be either an observed or a virtual unit. However, in some real problems the DM may wish to evaluate only existing units. In such cases, the non-convex value efficiency analysis model, suggested by Halme et al. (2014) is useful. The idea of the model is to construct convex preference cones and then use them to approximate the contours of the value function passing through the most preferred solution. The authors applied their model to evaluate the performance of bank branches in Helsinki, Finland.

\subsubsection{Dehnokhalaji et al. (2017)}

An important issue in DEA is to rank order efficient units. Based on the approach proposed by Dehnokhalaji et al. (2014) for constructing a total order for alternatives evaluated in terms of multiple criteria, Dehnokhalaji et al. (2017) developed a method for ranking units in DEA. They implemented and modified the radial model formulated by Dehnokhalaji et al. (2014) to obtain a non-radial measure, which is more stable in practice. The main idea of their model is to construct preference cones and preference polyhedra in the context of DEA and then find the distance of 
the unit under assessment to the convex cone/the polyhedral in terms of $\mathrm{L}-1$ norm. Then, according to the calculated distances, they were able to generate a full ranking of all units under consideration. Moreover, the accuracy of the results has been investigated through computational tests.

\subsection{Capturing preferences for inequality aversion: Karsu et al. (2018)}

Karsu et al. (2018) considered fairness problems, which occur widely in the public sector. In these problems the DM has both efficiency as well as equity or fairness concerns. The authors provided a procedure for ranking a discrete set of distributions (of income, wealth, health). In a societal setting, there is usually some initial preference information available regarding different distributions. Available preference information between some pairs of distributions is skillfully used to infer additional preference information. The authors proposed a tractable computational scheme for using the available preference information in order to derive a stronger ranking of distributions. This was accomplished via the use of convex cones. They provided some simulation results showing that their proposed method is computationally feasible for problems with small and medium sizes. They provided the first extensive work to incorporate preference information via convex cones into fairness problems.

\section{Application of convex preference cones for continuous/integer problems}

With some exceptions, the application of the convex cone theory to continuous multi-objective optimization problems or to integer programming problems is rather recent. We review a number of such studies.

\subsection{Use of convex cones in interactive multiple objective optimization: Prasad et al. (1997)}

Prasad et al. (1997) generalized the idea of convex cones to incorporate the idea how much an alternative lies above a convex cone. They developed the so-called $p$ cones concept and defined the $p$ cone efficiency, which measures the distance of an alternative from the region dominated by the convex cone. The smaller the obtained $p$ cone efficiency, the closer the alternative is to be dominated by the convex cone. Cone-dominated alternatives have a $p$ cone efficiency equal to zero. They proposed two procedures, namely, Acceleration and Early Termination, based on the obtained $p$ cone efficiencies, to reduce the required preference information. By extracting new preference information (within an interactive solution framework), they reduced the preference information requirements to improve the convergence of multi-objective linear programming procedures. 


\subsection{An interactive evolutionary algorithm to solve continuous and integer programming problems: Sinha et al. (2018)}

Sinha et al. (2018) introduced an interactive evolutionary algorithm to solve multiobjective continuous and integer programming problems. They assumed that the underlying value function is strictly increasing and quasi-concave. Since the Pareto optimal frontier is high-dimensional for such problems and visualizing it is very challenging, they let the DM guide the search towards the most preferred solution. On one hand, they eliminated non-preferred solution candidates by applying these cones, and on the other hand, they introduced extreme gradient directions to boost the evolutionary search. Borrowing from Fowler et al. (2010), they also constructed a cone-based merit (or fitness) function to measure the quality of the solution candidate. The direction and the merit function constructed based on the cones accelerates the convergence of their proposed algorithm. The approach successfully reached the most preferred solution for all test problems for different value functions.

\subsection{Multi-objective integer programming: Ramesh et al. $(1989,1990)$ and Lokman et al. $(2016,2018)$}

Early work in using convex cones to solve multi-objective integer programming problems is Ramesh et al. (1989, 1990). They developed a branch-and-bound scheme using convex cones. The tests are for Bi-criteria problems only. The approaches developed showed promise; however, were not capable of solving large-scale integer programming problems with the computing power available at the time.

In a recent paper we developed an exact interactive algorithm that finds the most preferred solution for multi-objective integer programming problems (Lokman et al. 2016). Again, we assumed that the DM's preferences are consistent with a quasiconcave value function. Based on the properties of quasi-concave value functions and pairwise preference information obtained from the DM, we generated constraints to restrict the implied inferior regions. An additional computational difficulty arises in these problems in characterizing the feasible region, as it becomes non-convex with the introduction of cone constraints. The algorithm continues iteratively and guarantees to find the most preferred solution for integer programs. We tested the performance of the algorithm on multi-objective combinatorial problems. Due to the inherent difficulty of integer programs, the increase in the number of non-dominated points, and the additional complexity of characterizing non-convex regions, the algorithm requires substantial computational effort for large-sized problems.

The computational difficulties of our 2016 algorithm served for us as a motivation to develop a computationally powerful algorithm, at the expense of a small (and controllable at a desired level) deterioration in solution quality (Lokman et al. 2018). Specifically, we approximated the most preferred solution of a multi-objective integer programming problem for any desired level of accuracy by establishing regions that are possibly inferior. As before, the DM's preferences were assumed 
to be consistent with an increasing quasi-concave value function. Using pairwise comparisons of the DM, we constructed convex cones and eliminated the inferior regions that are 'close' to being dominated by the cones in addition to the regions dominated by the cones. The performance of the algorithm was tested on a number of multi-objective combinatorial optimization problems. The performance of the algorithm is very good in terms of the quality of the solution found, the solution time, and the required number of pairwise comparisons. This demonstrates that it is a viable approach to solving multi-objective combinatorial optimization problems as well as general integer programming problems. For an application to multi-objective vehicle routing, see Tezcaner and Köksalan (2011, 2016), and to auctions Karakaya and Köksalan (2016).

\section{Conclusion}

This paper overviews the powerful idea and the literature behind the convex preference cones for solving different types of MCDM problems. The only input required from a DM is a number of pairwise preference comparisons, that is which one out of two alternatives is preferable. Convex preference cones have been found very useful in diverse applications, including multi-objective integer programming, evolutionary multi-objective optimization, and in generating a partial or total order for alternatives. An interesting recent generalization of the cone idea is by Karakaya et al. (2018).

We conclude with brief state-of-the art recommendations, what type of convex preference cones to form and how to use them. It is difficult to directly recommend some cone type, that is two-point, three-point etc., cones. Hence, we have on purpose been cautious in our recommendations. One thing is sure, however. Once we have the preference information (pairwise comparisons), we do want to generate all possible cones (to maximally use the available information), even though at some computational cost. But it is also possible to design the pairwise comparisons in suitable ways, so that they would, for example maximally support the formation of two-point cones. Such two-point cones have the advantage of simplicity and are efficient per question asked. We do not want too-many-point cones, but suitable threeor four-point cones are also effective in many situations. Including dummy alternatives in comparisons is good, because it helps enlarging the eliminated space, but may be criticized as being artificial.

Acknowledgements Open access funding provided by Aalto University. The authors wish to thank Professor Murat Köksalan, University of Michigan, Ann Arbor, for useful comments.

Open Access This article is distributed under the terms of the Creative Commons Attribution 4.0 International License (http://creativecommons.org/licenses/by/4.0/), which permits unrestricted use, distribution, and reproduction in any medium, provided you give appropriate credit to the original author(s) and the source, provide a link to the Creative Commons license, and indicate if changes were made. 


\section{References}

Charnes A, Cooper WW, Rhodes E (1978) Measuring the efficiency of decision making units. Eur J Oper Res 2:429-444

Deb K (2001) Multi-objective optimization using evolutionary algorithms. Wiley, New York

Dehnokhalaji A, Korhonen P, Köksalan M, Nasrabadi N, Wallenius J (2011) Convex cone-based partial order for multiple criteria alternatives. Decis Support Syst 51:256-261

Dehnokhalaji A, Korhonen P, Köksalan M, Nasrabadi N, Tezcaner D, Wallenius J (2014) Constructing a strict total order for alternatives characterized by multiple criteria. Nav Res Logist 61(2):155-163

Dehnokhalaji A, Hallaji B, Soltani N, Sadeghi J (2017) Convex cone-based ranking of decision-making units in DEA. OR Spectr 39:861-880

Fowler J, Gel E, Köksalan M, Korhonen P, Marquis J, Wallenius J (2010) Interactive evolutionary multiobjective optimization for quasi-concave preference functions. Eur J Oper Res 206:417-425

Halme M, Joro T, Korhonen P, Salo S, Wallenius J (1999) A value efficiency approach to incorporating preference information in data envelopment analysis. Manag Sci 45(1):103-115

Halme M, Korhonen P, Eskelinen J (2014) Non-convex value efficiency analysis and its application to bank branch sales evaluation. Omega 48:10-18

Hazen GB (1983) Preference convex unanimity in multiple criteria decision making. Math Oper Res 8(4):505-516

Karakaya G, Köksalan M (2016) An interactive approach for Bi-attribute multi-item auctions. Ann Oper Res 245:97-119

Karakaya G, Köksalan M (2018) Interactive approaches for Bi-objective problems with progressivelychanging solution sets. Int Trans Oper Res 25(3):1027-1052

Karakaya G, Köksalan M, Ahipasaoglu SD (2018) Interactive algorithms for a broad underlying family of preference functions. Eur J Oper Res 265(1):248-262

Karsu Ö (2013) Using holistic multicriteria assessments: the convex cones approach. In: Cochran J (ed) Wiley encyclopedia of operations research and management science. Wiley, New York, pp 1-14

Karsu Ö, Morton A, Argyris N (2018) Capturing preferences for inequality aversion in decision support. Eur J Oper Res 264(3):686-706

Köksalan M (1989) Identifying and ranking a most preferred subset of alternatives in the presence of multiple criteria. Nav Res Logist 36(4):360-372

Köksalan M, Sagala PN (1995) An approach and computational results on testing the form of a decisionmaker's utility function. J Multicriteria Decis Anal 4:189-202

Köksalan M, Taner OV (1992) An approach for finding the most preferred alternative in the presence of multiple criteria. Eur J Oper Res 60:52-60

Köksalan M, Karwan M, Zionts S (1984) An improved method for solving multiple criteria problems involving discrete alternatives. IEEE Trans Syst Man Cybern 14:24-34

Köksalan M, Karwan M, Zionts S (1986) Approaches for discrete alternative multiple criteria problems for different types of criteria. IIE Trans 18(3):262-270

Köksalan M, Karwan M, Zionts S (1988) An approach for solving discrete alternative multiple criteria problems involving ordinal criteria. Nav Res Logist 35(6):625-641

Korhonen P, Wallenius J, Zionts S (1984) Solving the discrete multiple criteria problem using convex cones. Manag Sci 30(11):1336-1345

Korhonen P, Moskowitz H, Wallenius J (1986) A progressive algorithm for modeling and solving multiple-criteria decision problems. Oper Res 34(5):726-731

Korhonen P, Soleimani-damaneh M, Wallenius J (2016) Dual cone approach to convex-cone dominance in multiple criteria decision making. Eur J Oper Res 249(3):1139-1143

Korhonen P, Soleimani-damaneh M, Wallenius J (2017) The use of quasi-concave value functions in MCDM: some theoretical results. Math Methods Oper Res 86(2):367-375

Lokman B, Köksalan M, Korhonen P, Wallenius J (2016) An interactive algorithm to find the most preferred solution of multi-objective integer programs. Ann Oper Res 245(1-2):67-95

Lokman B, Köksalan M, Korhonen P, Wallenius J (2018) An interactive approximation algorithm for multi-objective integer programs. Comput Oper Res 96:80-90

Malakooti B (1988) A decision support system and a heuristic interactive approach for solving discrete multiple criteria problems. IEEE Trans Syst Man Cybern 18(2):273-284 
Marquis J, Gel E, Fowler J, Köksalan M, Korhonen P, Wallenius J (2015) Impact of number of interactions, different interaction patterns, and human inconsistencies on some hybrid evolutionary multiobjective optimization algorithms. Decis Sci 46(5):981-1006

Olson D (1996) Decision aids for selection problems. Springer, New York, pp 81-95

Özpeynirci Ö, Özpeynirci S, Kaya A (2017) An interactive approach for multiple criteria selection problem. Comput Oper Res 78:154-162

Prasad SY, Karwan M, Zionts S (1997) Use of convex cones in interactive multiple objective decision making. Manag Sci 43(5):723-734

Ramesh R, Karwan M, Zionts S (1988) A theory of convex cones in multicriteria decision making. Ann Oper Res 16:131-147

Ramesh R, Karwan M, Zionts S (1989) Preference structure representation using convex cones in multicriteria integer programming. Manag Sci 35(9):1092-1105

Ramesh R, Karwan M, Zionts S (1990) An interactive method for bicriteria integer programming. IEEE Trans Syst Man Cybern 20(2):395-403

Sinha A, Malo P, Kallio M (2018) Convex preference cone-based approach for many objective optimization problems. Comput Oper Res 95:1-11

Taner OV, Köksalan M (1991) Experiments and a method for solving the discrete alternative multiple criteria problem. J Oper Res Soc 42:383-391

Tezcaner DÖ, Köksalan M (2011) An interactive algorithm for multi-objective route planning. J Optim Theory Appl 150:379-394

Tezcaner DÖ, Köksalan M (2016) An interactive approach for Bi-objective integer programs under quasiconvex preference functions. Ann Oper Res 244(2):677-696

Ulu C, Köksalan M (2001) An interactive procedure for selecting acceptable alternatives in the presence of multiple criteria. Nav Res Logist 48:592-606

Ulu C, Köksalan M (2014) An interactive approach to multicriteria sorting for quasiconcave value functions. Nav Res Logist 61:447-457

Zionts S, Wallenius J (1976) An interactive programming method for solving the multiple criteria problem. Manag Sci 22(6):652-663

Publisher's Note Springer Nature remains neutral with regard to jurisdictional claims in published maps and institutional affiliations. 